\title{
gGSS-API: a group enhanced generic security service
}

\author{
A.C.M. Hutchison \\ Data Network Architectures Laboratory, \\ University of Cape Town, \\ Private Bag, Rondebosch, ZA-7700 South Africa. \\ Telephone: +2721650 4058. Fax: +2721650 3726. \\ email: hutch@cs.uct.ac.za
}

\begin{abstract}
The Generic Security Service Application Programming Interface (GSS-API) offers a common interface to mechanism and protocol independent security. The contexts defined by GSS-API only support point-to-point interactions, and no support is provided for the interaction of multiple parties. This document describes group GSS-API (gGSS-API), an extension to support secure group contexts. As demonstration of one possible manner in which the group security features of such an extended API can be achieved, a sample protocol realization is outlined. Concerning deployment of gGSS-API, its use together with a secure IP multicast service is described as evidence that there are multiple ways in which, and layers at which, a group generic security service can be useful.
\end{abstract}

Keywords

Group security, GSS-API, generic security services

\section{INTRODUCTION}

Software supporting group interaction has received growing interest in recent years, and as commercial organizations have started embracing this technology so security for group applications has become a critical requirement. The Generic Security Service Application Programming Interface (GSS-API) (Linn, 1993; Linn, 1996) provides contexts as instances of secure communication. These contexts are currently limited to two-party interaction, taking no account of group scenarios. This paper proposes that these associations of GSSAPI should be enhanced to enable group (multi-party) contexts, in addition to the two party contexts currently supported. Such an extension, to create a group GSS-API (or gGSS-API) would enable deployment of GSS-API in environments where secure group interaction is required.

In principle it would be possible to construct a co-ordinating layer on top of the existing GSS-API, to establish and maintain a secure meta-context amongst multiple parties. This adds an extra level of indirection, however, and does not provide an integrated solution. 
A preferable approach, and that taken by gGSS-API, is to extend the existing GSS-API, allowing multiple parties to create group contexts with the same mechanism and protocol independence that the existing definition achieves.

Enabling group contexts in GSS-API requires several modifications to the API, and these are described. While mechanism independence is achieved, specific mechanisms (Hutchison and Bauknecht, 1996) can be used to achieve secure group contexts in the case where a shared group cryptographic key is established.

\section{OVERVIEW OF GSS-API}

Four design goals are outlined for GSS-API:

- Mechanism independence: The GSS-API defines only an interface to cryptographically implemented authentication and other security services. The definition is at a generic level and is independent of particular underlying mechanisms.

- Protocol environment independence: The GSS-API is independent of any protocol suites with which it is employed. This allows it to be used in a wide range of protocol environments.

- Protocol association independence: The GSS-API's security context is independent of communications protocol association constructs. This allows a single GSS-API implementation to be used by a variety of invoking protocol modules.

- Suitability to a range of implementation placements: GSS-API clients are not constrained to reside within any Trusted Computing Base (TCB) perimeter defined on a system where the GSS-API is implemented.

\subsection{Credentials}

A set of credentials is acquired by a particular application, and is used as a proof of identity for presentation to other processes. It is through the use of these credentials that a pair of communicating applications establishes a joint security context. A security context is described as a pair of GSS-API data structures that contain shared state information.

\subsection{Tokens}

To establish and maintain the shared information constituting a security context, certain GSS-API calls return a token. A token is a cryptographically protected, opaque data type. The responsibility for transferring tokens to a peer application is left with the caller of the GSS-API routine. Tokens can be distinguished as context level tokens, which are exchanged in order to establish and manage a security context between peers, and per message tokens which are exchanged (in conjunction with an established context) to provide protective security services for corresponding data messages. The transfer of tokens can be either in-band or out-of-band at the discretion of the GSS-API caller. 


\subsection{Stages of use}

The presentation of credentials and tokens can be summarized with an exposition of their stages of use:

- Credential acquirement is the phase during which a set of GSS-API credentials is established.

- Security context establishment occurs between a pair of communicating applications making use of their credentials. Tokens are returned by GSS-API calls and these are exchanged. As part of the security context establishment, the context initiator is authenticated to the responder (and may require that the responder is authenticated).

- Per message service invocation occurs according to the security context, and may apply integrity and data origin authentication (through calls to getMIC and verifyMIC functions ${ }^{*}$ at the sender and receiver, respectively) or confidentiality, integrity and data origin authentication (through calls to wrap and unwrap functions ${ }^{\dagger}$ at the sender and receiver, respectively).

- Security context deletion occurs at the completion of a communication session.

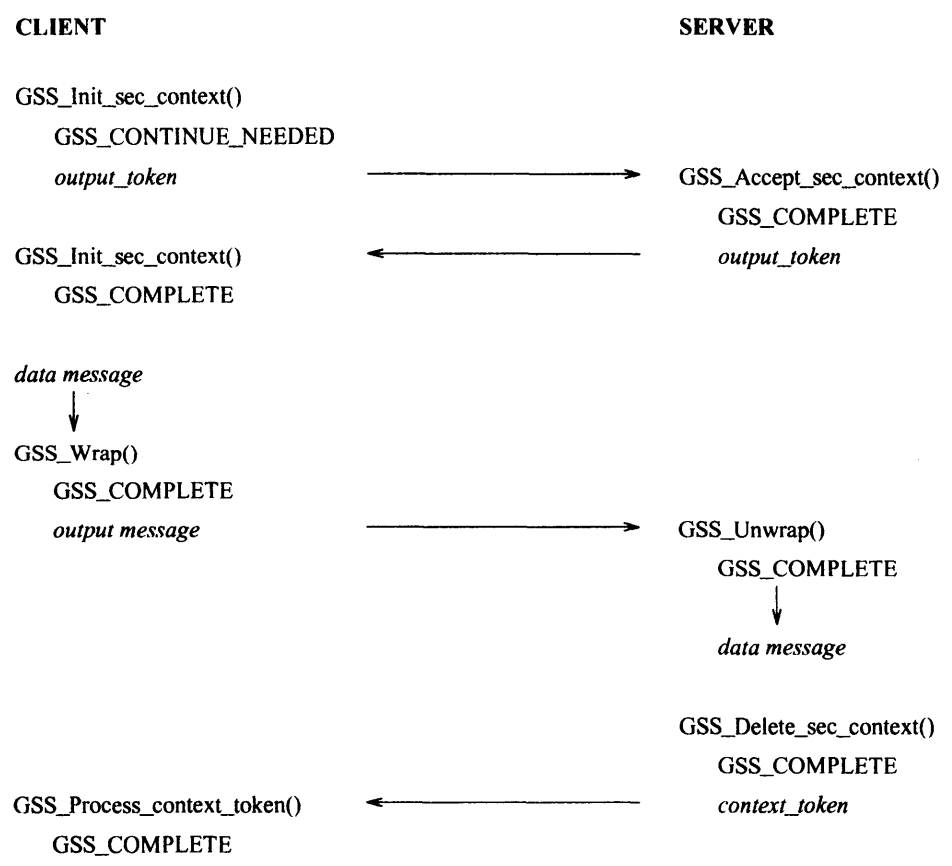

Figure 1 GSS-API example usage

\footnotetext{
${ }^{*}$ These are referred to as sign and verify functions in (Linn, 1993) but these terms are deprecated in current Internet Draft revisions (Linn, 1996).

†These are referred to as seal and unseal functions in (Linn, 1993) but these terms are deprecated in current Internet Draft revisions (Linn, 1996).
} 
Figure 1 shows the calls which might occur between a client and a server to establish a security context and transfer wrapped messages between them. GSS-API C bindings are proposed in (Wray, 1993). Design of a management interface has was considered in (McDermott and Kamens, 1994), and functions GSS_Add_Mechanism, GSS_Delete_Mechanism and GSS_Replace_Mechanism have been advanced for the dynamic addition, deletion and replacement of new mechanisms by privileged users.

Figure 2 presents a GSS-API implementation of Kerberos-like (Steiner, Neuman and Schiller, 1988) authentication. This example is provided as a reference point for the subsequent discussion on how GSS-API can be extended for group protocols.

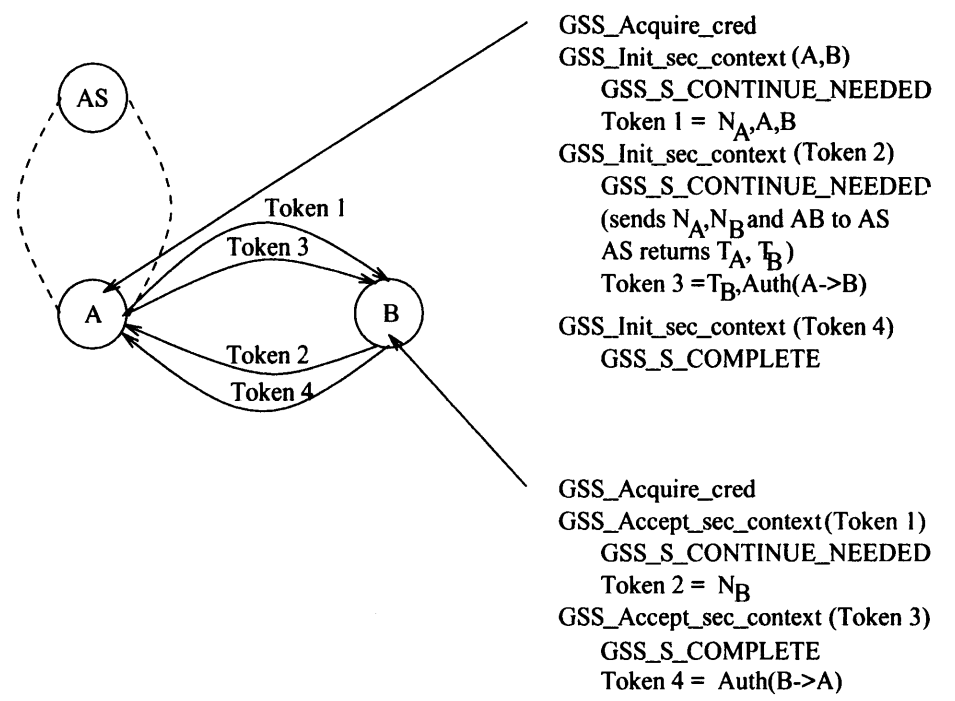

Figure 2 GSS-API implementation of Kerberos like authentication

\section{3 gGSS-API: GROUP SUPPORT EXTENSIONS}

In the context of group support, it is conceivable that the use of multicasting $\ddagger$ would be the most attractive and efficient means of transferring tokens to $n$ peer applications. But since one of the design goals identified was 'protocol association independence' the group solution should not necessarily make this assumption.

It is considered that in the context of extending GSS-API for group support it is still necessary to have an originator. In order to initiate a multiparty security context, one or more exchanges with each group member is required. The exact number and manner of exchanges between a group originator and group members is not prescribed by the API but in Section 3.3 one possible implementation is described.

By mapping the specifics of these techniques to gGSS-API tokens, and extending certain GSS-API functions to accommodate group contexts, a generic security service for groups can be achieved.

\footnotetext{
$\ddagger$ Whereby a single message is sent to a group of recipients, in the manner of a selective broadcast.
} 


\subsection{Changes to context level calls}

In the following paragraphs specific changes required for the gGSS-API functions used to establish a security context are described.

\section{Principal naming}

When a multi-party security context is required, a means of listing the communicating parties in GSS_Init_sec_context is required. Similarly, it is necessary to return multiple tokens which also require identification. Returning tokens in an order corresponding to the principal naming allows the initiator to demarcate the tokens. Distribution of the returned tokens remains the responsibility of the calling process. The calling process is also responsible for collecting (multiple) reply tokens and submitting these to subsequent GSS_Init_sec_context calls.

\section{Channel bindings}

The channel_bindings used in two party communication can be extended in at least two ways. The first is to replace the acceptor_address with a group address, if an address such as Internet Class D $\S$ can be supplied. The second approach, in a non-multicast type environment, would be to provide a list of channel bindings associated with each of the supplied communication partner identities and in this manner to build specific channel information into each of the returned tokens. This can be achieved by creating a list of acceptor addresses (replacing the gss_buffer_desc type with with the gss_OID_set_desc type). This provides greater security than simply giving a single multicast group address, but requires more coordination and necessitates individual message delivery or the addition of per-member information into a multicast message.

\subsection{Changes to per-message calls}

The gGSS-API calls which are called on a per-message basis enable a sender to deliver a signed and/or 'wrapped' message to a receiver who is able to verify and/or 'unwrap' the message. The goal of the group extension concerning these calls, is that the functions should also provide security for messages destined for multiple recipients.

The invocation of GSS_GetMIC and GSS_Wrap is determined by the manner of group key which is applied. If a single group session key is being shared amongst the communication partners and a single signed or wrapped message is distributed to other parties then no changes to the parameters of the per-message calls are required. If different keys are used between different communication partners (which may be the case according to the implementation) then it may be necessary to accept a list of output messages from the GSS_GetMIC and GSS_Wrap routines with different messages bound for different recipients. No changes are required in the GSS_VerifyMIC and GSS_Unwrap routines since the manner of decoding is unaffected by the number of messages returned by the encoding functions.

Processing of GSS_VerifyMIC and GSS_Unwrap may be different in a group context, for example if a session key is encrypted with each recipient's public key then the

§Class D IP addresses are those with ' 1110 ' as their high-order four bits. In Internet 'dotted decimal' notation, host group addresses range from 224.0 .0 .0 to 239.255 .255 .255 . 
recipient has to be able to find the offset of the expression relevant to them in a list of expressions.

\subsection{Group cryptographic keys and authentication}

Having introduced an extended gGSS-API for the purpose of providing secure group contexts, we now turn to the actual realization of the additional group security features. The whole generic security service philosophy is that its use is implementation independent, but in proposing a new aspect of support (for groups) it is also important to demonstrate how an underlying implementation could be provided.

For secure group interaction, a set of group authentication protocols is necessary. An overview of such protocols is given in (Hutchison and Bauknecht, 1996). In particular, these protocols for secure group authentication and key distribution can be distinguished by complete or selective interaction amongst group members and can also be implemented

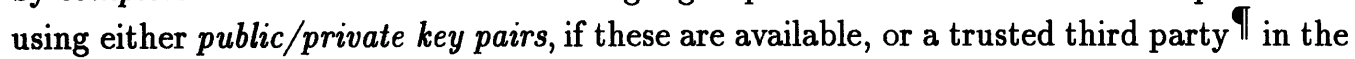
form of an authentication server. In a complete authentication interaction, each member of a group authenticates each other group member. Selective authentication refers to cases where interaction is with a single group custodian (or 'gatekeeper') only, or cases where a member joining a group authenticates with all existing members of the group (but these members do not authenticate one another again). Depending on the security requirements, different authentication models may apply. In subsequent discussion in this paper a complete group authentication is applied.

To conduct group authentication via gGSS-API there has to be a mapping from an authentication protocol to the gGSS-API tokens. We can use a complete group authentication protocol, based on the presence of an authentication server, to illustrate a mapping from protocol to tokens. The process of mapping such a group authentication protocol is analogous to the mapping of two-party Kerberos-like authentication which resulted in Figure 2.

Using the notation of:

- $\rightarrow$ to represent a unicast from one principal to another

- $G$ to represent all members of the group

- AS to represent an authentication server

- $N_{X}$ to represent a random number (nonce) generated by principal $\mathrm{X}$

- $T_{X}$ to represent a ticket (cryptographic token) for principal $X$ and

- $\operatorname{Auth}(\mathrm{X} \longrightarrow \mathrm{Y})$ to represent a token containing information by which principal $\mathrm{X}$ can authenticate itself to principal $Y \|$

we can generalize a complete group authentication and key distribution protocol as consisting of the following rounds:

IOr more precisely ' $(n+1)$-th party' with $n$ participants.

IDescription of the complete structure of the tickets and authenticator tokens is beyond the scope of this paper. 

1. $A \rightarrow X: \quad N_{A}, G$
$(\forall X \in(G-\{A\}))$
2. $X \rightarrow A: \quad N_{X}$
$(\forall X \in(G-\{A\}))$
3. $A \rightarrow A S: N_{X}, G$
$(\forall X \in G)$
4. $A S \rightarrow A: T_{X}$
$(\forall X \in G)$
5. $A \rightarrow X: \quad T_{X}, \operatorname{Auth}(A \rightarrow X), N_{Y}$
$(\forall X \in(G-\{A\}), \forall Y \in(G-\{A, X\}))$
6. $X \rightarrow A: \operatorname{Auth}(X \rightarrow Y)$
$(\forall X \in(G-\{A\}), \forall Y \in(G-\{X\}))$
7. $A \rightarrow X: A u t h(Y \rightarrow X)$
$(\forall X \in(G-\{A\}), \forall Y \in(G-\{X, A\}))$

In Figure 3 a mapping of this initiator coordinated complete group authentication protocol to a gGSS-API type implementation is shown. The gGSS-API calls of A (coordinator) and $B$ (group member) are shown. The calls of $C$ and $D$ are analogous to those of $B$. The token contents are given as an indication of how the rounds of a protocol such as that above can be realized in a gGSS-API context.

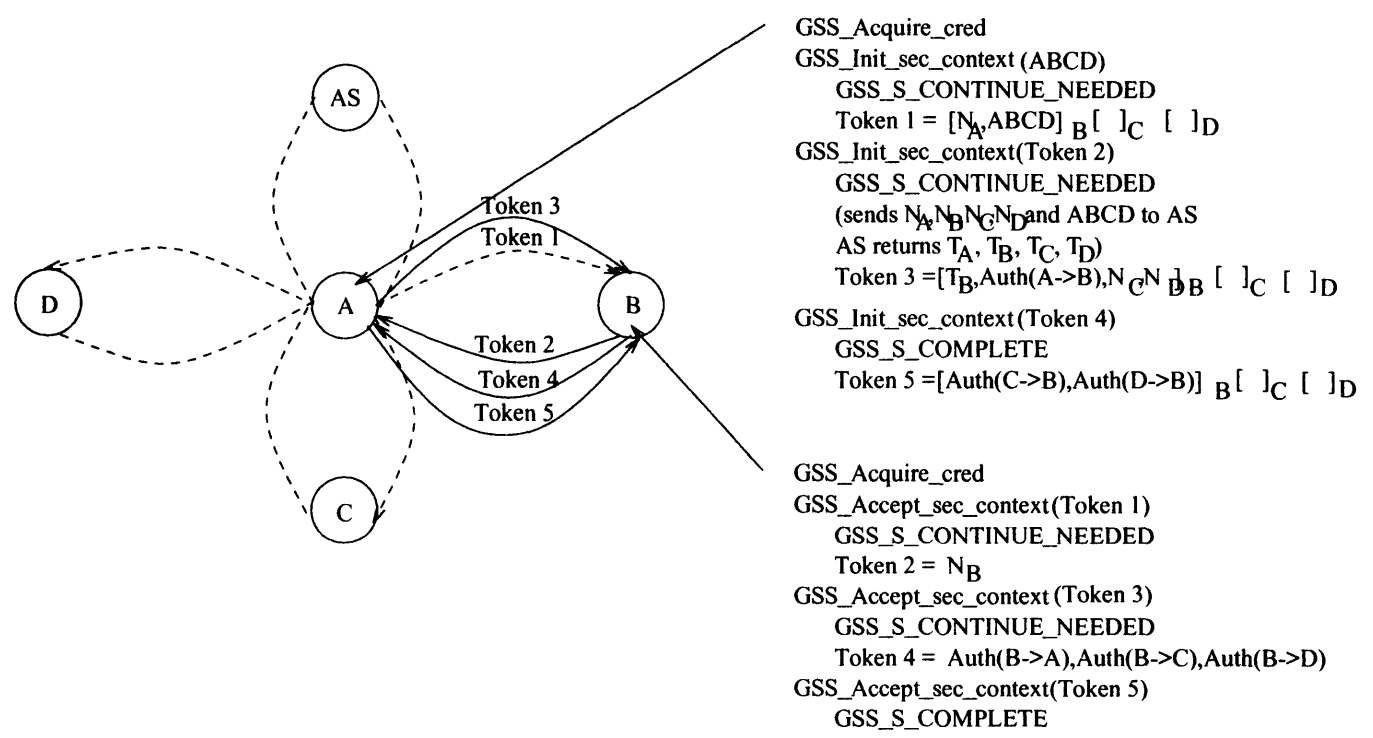

Figure 3 gGSS-API calls in establishing a group context

\section{4 gGSS-API DEPLOYMENT: SECURE IP MULTICAST}

At the start of this paper it was implied that (group) security is typically implemented at the application level. To demonstrate that gGSS-API can be deployed to complement lower layer security deployment, its use together with a secure IP multicast is described here.

IP multicast allows point-to-multipoint communication via a Class D multicast address which identifies a destination group. Figure 4 shows a conceptual layering together with 


\begin{tabular}{|c|c|}
\hline \multicolumn{2}{|c|}{ Application } \\
\hline $\begin{array}{l}\text { Reliable Stream } \\
\text { (TCP) }\end{array}$ & $\begin{array}{l}\text { User Datagram } \\
\text { (UDP) }\end{array}$ \\
\hline \multicolumn{2}{|c|}{$\begin{array}{l}\text { Internet } \\
\text { (IP) }\end{array}$} \\
\hline \multicolumn{2}{|c|}{ Network Interface } \\
\hline
\end{tabular}

OSI 7 (Presentation)

OSI 4 (Transport)

OSI 3 (Network)

OSI 1-2 (Physical/Data Link)

Figure 4 Conceptual communication layering

associated OSI layers (Comer, 1992): a secure IP multicast suggests that at the IP layer, multicast (and unicast) IP messages can be processed to achieve confidentiality, integrity, replay detection, etc. according to a caller-specified quality of protection (QoP).

The advantage of providing security mechanisms at the IP level is that it is then not necessary for each application requiring secure communication to go about implementing this. The desire for and appropriateness of security being implemented at this level has been advocated in discussions of IPng (Bellovin, 1994) and also in (Cheng, Garay, Herzberg and Krawczyk, 1995) where it is argued that the IP layer is an appropriate place to secure data since the secrecy and integrity of data can be protected in an internetwork environment without affecting higher-layer protocols and applications. Another argument in favor of security at the IP level is that IP headers can be protected using cryptographic techniques with the result that packet filtering can be done based on authentic information (which is very useful for Internet firewalls).

For an IP level secure multicast group implementation, one or more cryptographic keys would be provided to the multicast service by the calling application. Key origination and distribution are typically outside the scope of a secure IP multicast mechanism but a library like gGSS-API complements IP level security by allowing groups of application level processes to establish group keys which can then be used in conjunction with a secure IP multicast.

The approach taken is that the key(s) is/are provided to the IP service interface, together with an indication of the QoP desired. According to the QoP requested, packets emanating from the host to the group addressed will be secured using one of the group keys provided. Similarly, packets received from a particular group will be processed in accordance with the required QoP.

Figure 5 shows the interaction of a secure IP multicast and gGSS-API, and how they can be integrated to provide application programmers with a secure environment which requires very little effort on their part if the IP multicast can be secured using the cryptographic keys which multicast group members hold. The gGSS-API is used amongst the processes $P_{1}, P_{2}, \ldots, P_{n}$ to authenticate each other and distribute one or more group keys (represented by $K_{G}$ ) which is/are then passed to the secure IP multicast layer allowing the IP module to perform the security processing function on packets leaving from and arriving at process sockets. 


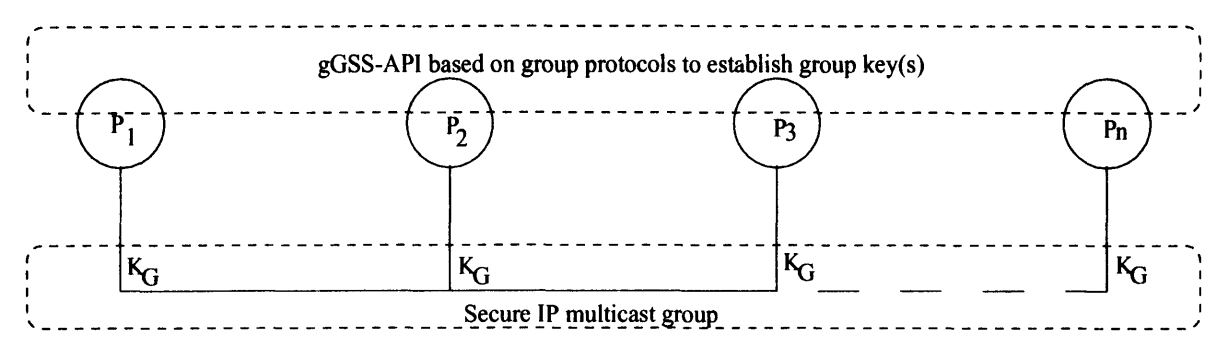

Figure 5 Interaction of gGSS-API and secure IP multicast

The steps which an application program goes through to implement this integrated solution to the problem of secure group communication are the following:

- Call gGSS-API to perform authenticated key distribution. This results in all processes holding the group key(s).

- Initialize socket communication for multicast.

- Initialize socket communication for secure multicast. This includes the provision of quality of protection parameters.

- Perform group multicast communication using the usual UDP sendto and recvfrom functions for IP multicast.

This example of gGSS-API deployment shows that in addition to native usefulness at the application layer, a group generic security service can be used when group authentication and key distribution are conducted at a higher layer. In this model group cryptographic information is passed to lower layers where support for a service like secure IP multicast is provided.

\section{CONCLUSION}

GSS-API is a well defined, mechanism and protocol independent, solution to key distribution and authentication problems. The use of GSS-API is good for application developers since the actual realization of the GSS-API can be substituted transparently. gGSS-API, an extension of GSS-API to provide group support, enables $n$ communicating parties to manage secure interaction. While introducing group functionality, it still retains support for all the usual two-party features of the original GSS-API and so is backwards compatible.

gGSS-API achieves the same goal that the security service achieved for two party interaction: freeing the application developer from having to do a security implementation and neatly abstracting the security processing - requiring only that applications transfer opaque tokens. The 'add-on' that gGSS-API provides, is that group support is provided and this is seen as increasingly important with the growing entrenchment of groupware and CSCW type applications. The interaction of gGSS-API with a secure IP multicast was described as an example of gGSS-API deployment spanning conceptual security implementation layers: its usefulness is not limited to the application layer.

Current work comprises implementation of a prototype library which provides a re- 
alization of the proposed group support. Consideration is also being given to whether gGSS-API could be used in a Java-like context to provide security for groups of applets interacting to provide CSCW type functionality.

\section{REFERENCES}

Bellovin, S. (1994) RFC1675: Security Concerns for IPng. ATET Bell Laboratories Cheng, P-C., Garay, J., Herzberg, A. and Krawczyk, H. (1995) Design and Implementation of Modular Key Management Protocol and IP Secure Tunnel on AIX. Proceedings of the 5th USENIX UNIX Security Conference, Salt Lake City, Utah.

Comer, D.E. (1992) Internetworking with TCP/IP: Principles, Protocols and Architecture. Prentice-Hall, Englewood Cliffs, New Jersey, 1992.

Hutchison, A. and Bauknecht, K. (1996) Cryptographic Key Distribution and Authentication Protocols for Secure Group Communication. Proceedings of the Twelfth International Information Security Conference (IFIP/SEC '96), Samos, Greece

Linn, J. (1993) RFC1508: Generic Security Service Application Program Interface. Geer Zolot Associates.

Linn, J. (1996) Generic Security Service Application Program Interface, Version 2. Internet Draft Document <draft-ietf-cat-gssv2-08.txt> (Work in Progress). Open Vision.

McDermott, E. Kamens, J. (1994) GSS-API Extensions for Multi-Mechanism Support. Draft Document.

Steiner, J., Neuman, C. and Schiller, J. (1988) Kerberos: An Authentication Service for Open Network Systems. Proceedings of USENIX Winter Conference.

Wray, J. (1993) RFC1509: Generic Security Service API: C-bindings. Digital Equipment Corporation.

\section{BIOGRAPHY}

Andrew Hutchison obtained an MSc in Computer Science from the University of Cape Town, South Africa in 1991, and a PhD in Computer Science from the University of Zürich, Switzerland in 1996. While studying in Switzerland he worked at the IBM Zürich Research Laboratory. He is currently a Senior Lecturer in the Department of Computer Science at the University of Cape Town. His research interests include computer networks, network security and performance evaluation. 